\title{
Blockchain Technology and Its Applications
}

Andy Wang, College of Applied Sciences and Arts, awang@ siu.edu

Keywords: Blockchain, Emerging technology, Assessment.

Blockchain technology was created for the digital cryptocurrency bitcoin in 2008. However, its applications are much wider than alternative currency and it is poised to be "the next big thing" in applied sciences. Marc Andreessen called Blockchain "one of the most important technologies since the advent of the Internet." In a nutshell, Blockchain is a distributed database that provides an unalterable public record of digital transactions. It can be viewed as a distributed digital ledger containing a chain of blocks information, where each block is identified by a cryptographic signature. These blocks are all back-linked; that is, they refer to the signature of the previous block in the chain, and that chain can be traced all the way back to the very first block created. As such, the Blockchain contains an un-editable record of all the transactions made. The transparent and decentralized nature of the Blockchain network enables the development of a non-refutable, and unbreakable record of data, which is the fundamental feature to many applications, such as insurance, finance, fraud detection, copyright protection, smart contracts, identity management, ecommerce and healthcare. Even in higher education, Blockchain technology can help track student credentials and achievements in a cheap, secure, reliable and public way.

One indicator of an important emerging technology is the strength of funding. It is interesting to notice that various funding sources are considering to "invest" in Blockchain technology, including venture capitals, various foundations, and federal agencies. Both the Defense Advanced Research Projects Agency (DARPA) of the U.S. Department of Defense (DoD) and NATO have put out requests for military-related apps built on Blockchain technology. DARPA wants to leverage Blockchain technology to create a "Secure Messaging Platform" to transfer messages via a secure decentralized protocol that will be secured across multiple channels, mapping the business logic of the DoD ecosystem onto a network of known entities using distributed ledgers. The NATO Communications and Information Agency is looking for proposals with transformational, state-of-the-art technology solutions in support of NATO C4ISR and cyber capability requirements as well as other military applications of Blockchains, including application of Blockchain technology to military logistics, application of Blockchain technology to procurement and finance.

Blockchain provides an increasingly popular means for education as well. The most notable example is the Learning As Earning (LAE) initiative proposed by the Institute for the Future (IFTF) and the ACT Foundation. The central idea of this initiative is the concept of Edublocks, which is similar to "credit hours" we are currently using to record and assess student learning. In addition to track academic learning activities, Edublocks can measure and record informal learning as well, such as diversity and inclusiveness trainings, RSO (Registered Student Organization) events, competitions, research presentations, externship experience, community service and so on. A chain of Edublocks forms a distributed ledger, or ePortfolio, for students to get credit for learning that happens anywhere anytime, on- or off-campus. The collection of all Edublocks a student earned during her study forms an electronic portfolio at her graduation 
containing all of the "earned income" - various skills.. The student can use this portfolio for job interviews, or as an important reference for employers to hire this student. The academic advisor in a Learning As Earning institution will focus on helping students to earn the most "incomes" in their portfolios. The benefits of this learning ledger include course assessment, curriculum improvement, and financial aid. It is important to note that this whole Learning As Earning idea is built on Blockchain technology.

Blockchains enable all parties to improve trust and transparency among various stakeholders in high education, including students, faculty, administrative staff, alumni, and employers. As electronic portfolios evolve from information repositories to robust tools for showcasing student learning, the next generation of assessment tools, measuring program quality and student learning outcomes, will be built using Blockchain technology. The paper discusses the state of the art in Blockchain technology and its applications, focusing on applications in higher education. 